\title{
Association between lutein intake and lung function in adults: the Rotterdam Study
}

\author{
Debora Melo van Lent ${ }^{1,2}$, Elisabeth T. M. Leermakers ${ }^{1}$, Albert Hofman ${ }^{1,3}$, Bruno H. Stricker ${ }^{1,4,5}$, \\ Guy G. Brusselle ${ }^{1,6,7}$, Oscar H. Franco ${ }^{1}$, Lies Lahousse ${ }^{1,6,7}$ and Jessica C. Kiefte-de Jong ${ }^{1,8_{*}}$ \\ ${ }^{1}$ Department of Epidemiology, Erasmus MC University Medical Center, 3000 CA, Rotterdam, The Netherlands \\ ${ }^{2}$ Deutsches Zentrum für Neurodegenerative Erkrankungen, 53175 Bonn, Germany \\ ${ }^{3}$ Department of Epidemiology, Harvard T.H. Chan, Boston, MA 02115, USA \\ ${ }^{4}$ Department of Internal Medicine, Erasmus MC University Medical Center, 3000 CA, Rotterdam, The Netherlands \\ ${ }^{5}$ Inspectorate of Health Care, Utrecht 3521 AZ, The Netherlands \\ ${ }^{6}$ Department of Respiratory Medicine, Erasmus MC University Medical Center, 3000 CA, Rotterdam, The Netherlands \\ ${ }^{7}$ Department of Respiratory Medicine, Ghent University Hospital, 9000 Ghent, Belgium \\ ${ }^{8}$ Leiden University College, 3595 DG The Hague, The Netherlands
}

(Submitted 2 August 2016 - Final revision received 16 December 2016 - Accepted 25 January 2017)

\section{Abstract}

Lutein, a fat-soluble carotenoid with antioxidant properties, may have an effect on respiratory health. However, the evidence is inconsistent. We aimed to cross-sectionally investigate the association between lutein intake and lung function by measuring forced expiratory volume in $1 \mathrm{~s}\left(\mathrm{FEV}_{1}\right)$, forced vital capacity (FVC) and $\mathrm{FEV}_{1} / \mathrm{FVC} \%$ in adults (aged $45-79$ years). We included 4402 participants from the Rotterdam Study, a prospective cohort study in The Netherlands. Lutein intake was assessed using a validated FFQ. Lung function was assessed using spirometry around the same time point as the dietary assessment. No independent association was found between lutein intake and $\mathrm{FEV} \mathrm{V}_{1}(-12 \cdot 17(95 \% \mathrm{CI}$ $-34 \cdot 21,9 \cdot 87) \mathrm{ml}$ per sD increase in lutein) after adjustment for age, sex, height, cohort effect, ethnicity, education, weight, total daily energy intake, smoking status, physical activity, and intakes of fatty acids, dietary fibre, alcohol, $\beta$-carotene, $\beta$-crypotoxanthin, lycopene and zeaxanthin. There was also no association between lutein and $\mathrm{FVC}$ or $\mathrm{FEV}_{1} / \mathrm{FVC} \%$. However, after stratification by smoking status, lutein intake was significantly associated with lower $\mathrm{FEV}_{1} / \mathrm{FVC} \%$ in current smokers $(-1.69$ (95\% CI $-2.93,-0.45) \%$ per sD increase of lutein) independent of other carotenoids. The present study does not support an independent association between lutein intake and lung function in adults. However, future studies should focus on the potential inverse association between high lutein intake and lung function in specific risk groups such as smokers.

\section{Key words: Lutein: Carotenoids: Antioxidants: Lung function: Adults: Elderly}

Chronic obstructive pulmonary disease (COPD) is currently the third cause of death worldwide ${ }^{(1)}$. Interestingly, poor lung function is an important predictor of mortality in patients with COPD and in the general population ${ }^{(2-4)}$.

Several studies have focused on evaluating the impact of nutritional therapy in COPD patients ${ }^{(5)}$; however, it is unclear how specific dietary components may influence lung function ${ }^{(6)}$.

Lutein is a carotenoid without vitamin A capacities, but with powerful antioxidant capacities ${ }^{(7)}$. The main sources of lutein are kale, spinach and collards ${ }^{(8)}$. Furthermore, lutein is well known for its antioxidant effect in the eye, where it protects the retina from inflammation and oxidative stress ${ }^{(7)}$. With this in mind, it may be the case that lutein has the same protective function in the lungs; counteracting a retardation in pulmonary function.
It is hypothesised that carotenoids, including lutein, can protect the airways from inflammation-induced damage. Schunemann et al. reported in both their studies (cross-sectionally and prospectively) a strong antioxidant effect of lutein (serum and dietary intake) on lung function ${ }^{(9,10)}$. It has been suggested that inadequate dietary intake of antioxidants is associated with the development of respiratory diseases ${ }^{(11,12)}$.

The association between lutein intake and respiratory health has recently been reviewed ${ }^{(13)}$, and very few studies in adult populations have been carried out ${ }^{(9,10,14-16)}$. However, these studies combined lutein with zeaxanthin and did not extensively adjust for confounders or comprised selected populations (e.g. only in women, young adults or patients with COPD). To date, there are no recommended levels of intake for lutein;

Abbreviations: COPD, chronic obstructive pulmonary disease; $\mathrm{FEV}_{1}$, forced expiratory volume in 1 s; FVC, forced vital capacity; RS-I-III, Rotterdam Study cohorts I-III.

* Corresponding author: J. C. Kiefte-de Jong, fax +31 107044 657, email j.c.kiefte-dejong@erasmusmc.nl 
therefore, from a public health perspective, it is important to determine whether recommendations are required. We aimed to cross-sectionally evaluate the effect of lutein intake on lung function as measured by forced expiratory volume in $1 \mathrm{~s}\left(\mathrm{FEV}_{1}\right)$, forced vital capacity (FVC) and the $\mathrm{FEV}_{1} / \mathrm{FVC} \%$, in adults from a general population (aged 45-79 years), taking into account socio-demographic, lifestyle and nutritional factors.

\section{Methods \\ Study design}

The present study was conducted with data from the Rotterdam Study, an ongoing, prospective, population-based cohort study since 1990, including adults aged 45 years or older living in the well-defined district Ommoord, a neighbourhood of Rotterdam, The Netherlands ${ }^{(17)}$. The Rotterdam Study was approved by the Medical Ethics Committee of the Erasmus University, and written informed consents were collected from all participants ${ }^{(17)}$. The original aim of the Rotterdam Study was to investigate factors related to frequently occurring diseases in the elderly, as this group is particularly vulnerable to diseases ${ }^{(17,18)}$. Further information about the objectives and study design of the Rotterdam Study itself is described elsewhere $^{(17)}$.

The baseline visit of the first Rotterdam Study cohort, including 7983 participants aged 55 years or older, was completed between 1990 and 1993 (Rotterdam Study cohort I (RS-I)-visit 1). From 2000 to 2001, the cohort was extended with a second cohort of 3011 individuals, who are now 55 years old or have moved to Ommoord district (RS-II). From 2006 to 2008, a third cohort of 3932 individuals aged 45-54 years living in Ommoord (RS-III) was added ${ }^{(17)}$. Participants were invited for follow-up measurements every 3-4 years.

\section{Study participants}

Data from the present study were collected from participants attending the fifth visit of the original cohort (RS-I-visit 5; 2009-2011; $n$ 2140), the third visit of the second cohort (RS-II-visit 3; 2011-2012; $n$ 1887) and the second visit of the third cohort (RS-III-visit 2; 2012-2013; $n$ 3000). We excluded participants for whom dietary intake data were not available ( $n$ 1337), participants who had an abnormal total energy intake below $2092 \mathrm{~kJ}$ ( $500 \mathrm{kcal}$ ) or above $20920 \mathrm{~kJ}$ ( $5000 \mathrm{kcal})(n$ 158) and participants who did not have an interpretable spirometry ( $n$ 1130). Thus, we included data from 4402 individuals in these analyses. A flow chart is given in Fig. 1.

\section{Dietary intake of lutein}

For RS-I-visit 5 (2009-2011), RS-II-visit 3 (2011-2012) and RS-III-visit 1 (2006-2008), self-reported dietary intake was assessed using a FFQ. Participants were asked to fill in a FFQ at home to report their nutritional intake in the past year. The FFQ was a comprehensive, 389-item, semi-quantitative questionnaire based on an existing validated FFQ developed for
Dutch adults ${ }^{(19,20)}$. The FFQ included questions such as the frequency of consumption of food items over the last month, the amount and type of food item and preparation methods. Portion sizes in $\mathrm{g} / \mathrm{d}$ were estimated using standardised household measures ${ }^{(21)}$. Dietary data were converted into nutrient intakes (including daily lutein intake and total energy intake) using the Dutch Food Composition Tables of 2006 and $2011^{(22,23)}$.

\section{Lung function}

The main outcomes assessed in the present study were the $\mathrm{FEV}_{1}, \mathrm{FVC}$ and $\mathrm{FEV}_{1} / \mathrm{FVC} \%$. Trained personnel measured lung function using Master Screen ${ }^{\circledR}$ PFT Pro (CareFusion) according to the American Thoracic Society (ATS)/European Respiratory Society (ERS) guidelines ${ }^{(24)}$. To ensure reproducibility, multiple efforts were required. The values of the best acceptable effort were used for analyses. Spirometry tests that did not meet ATS/ERS acceptability and reproducibility criteria were classified as 'not interpretable'.

\section{Confounders}

Before the baseline research centre visit, home interviews were conducted by trained interviewers. Thereafter, participants were invited for clinical examination and laboratory tests at the research centre. At the baseline visit of the original cohort (RS-I-visit 1 1990-1993), data of multiple factors were collected. For the present analyses, level of education and ethnicity of the baseline visit were used as proxies for education and ethnicity of the fifth visit (RS-I-visit 5; 2009-2011). Level of education was divided into two groups - low (primary education or less) and high (levels above primary education). Ethnicity of participants was based on the ethnic background of the participants' grandparents, and was divided into Caucasian and nonCaucasian. Physical activity was measured in metabolic equivalent task-h per week, which was a combination of questions on sport, walking, cycling and gardening. Smoking status was divided into three categories - never smoker, former smoker and current smoker. Data on the following confounders used for the analyses were collected from the fifth visit itself (RS-I-visit 5; 2009-2011): age, sex, height, weight, total daily energy intake, physical activity and smoking status. In addition, other potential dietary confounders were collected by the FFQ: total fat intake, ratio of $n-3: n-6$ fatty acids (N3:N6), and intakes of fibre, alcohol, $\beta$-carotene, $\beta$-cryptoxanthin, lycopene and zeaxanthin.

At the baseline visit of the second cohort (RS-II-visit 1; 2000-2001), data on education and ethnicity were collected in the same way as for the baseline visit of the original cohort and were used as proxies for education and ethnicity of the third visit (RS-II-visit 3; 2011-2012). Ethnicity was based on the ethnic background of the participants' parents, and was divided into the same categories as the RS-I-visit 1 . The other factors were collected from the third visit itself.

Data on education, ethnicity, physical activity and dietary intake of the first visit of the third cohort (RS-III-visit 1; 2006-2008) were also collected according to the above-mentioned methods and 


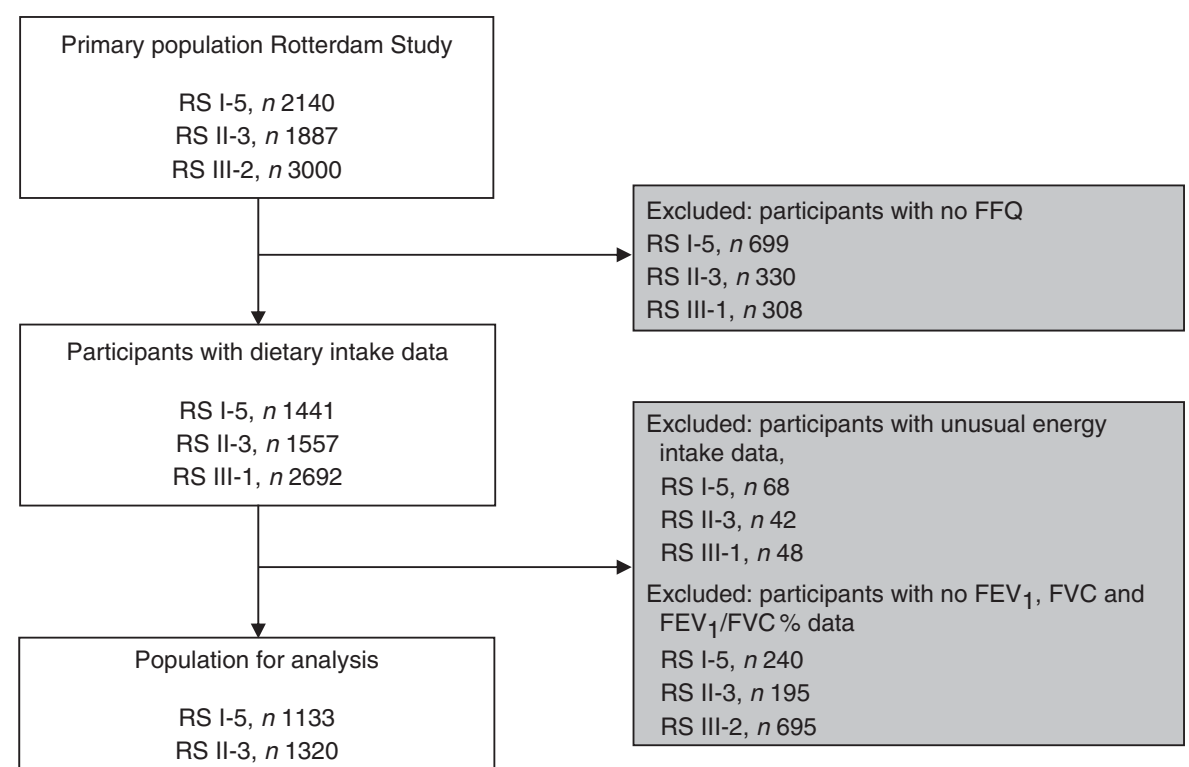

Fig. 1. Flow chart of the participants included in the study ( $n$ 4402). RS-I-III-1-5, Rotterdam Study cohorts I-III-visits $1-5$; FEV 1 , forced expiratory volume in $1 \mathrm{~s}$; FVC, forced vital capacity.

used as proxies for education, ethnicity and dietary intake data of the second visit (RS-III-visit 2; 2012-2013). The other factors were collected from the second visit itself.

\section{Statistical analysis}

We used linear regression analysis to estimate cross-sectionally the effect of $1 \mathrm{sD}$ increase in dietary lutein intake (continuous) on the change in $\mathrm{FEV}_{1}(\mathrm{ml}), \mathrm{FVC}(\mathrm{ml})$ and $\mathrm{FEV}_{1} / \mathrm{FVC} \%$. We also tested the relationship for non-linearity using natural splines (online Supplementary Fig. S1) ${ }^{(25)}$. There was no evidence for a non-linear relationship, but as no recommended daily intake of lutein exists, we decided to categorise dietary lutein intake into four quartiles. As there is no European reference data for lutein intake available, the second quartile (1.9-3.2 mg) was chosen as a reference group, as this quartile was in agreement with the average daily intake of lutein for males $(2 \cdot 0-2.3 \mathrm{mg})$ and females $(1 \cdot 7-2 \cdot 0 \mathrm{mg})$ in the $\mathrm{USA}^{(26)}$.

Confounders were selected on the basis of published literature and a $10 \%$ change in the effect estimate of the association between dietary lutein intake and indices of lung function, as described by Mickey \& Greenland ${ }^{(27)}$. Lutein and other dietary covariates were adjusted for total energy intake, using the residual method ${ }^{(28)}$. Thereafter, analyses were performed with adjustment for social-demographic factors (age, sex, height, cohort, ethnicity and education) in the analyses labelled as model 1. Model 2 was additionally adjusted for lifestyle factors: weight, total daily energy intake, total fat intake, N3:N6, fibre intake, alcohol intake, smoking status and physical activity. To adjust for other carotenoids, model 3 included additional adjustment for intakes of $\beta$-carotene, $\beta$-cryptoxanthin, lycopene and zeaxanthin. $\alpha$-Carotene could not be included in this model because of multicollinearity (the correlation between $\alpha$-carotene and $\beta$-carotene was 0.96).
We tested for significant interactions $(P<0 \cdot 10)$ between lutein and sex, age, BMI $\left(\mathrm{BMI}=\right.$ weight $/$ height $\left.^{2}\right)$, smoking status, previous diagnosis of lung cancer, asthma and COPD. Subgroup analyses were conducted for smoking and BMI, as a significant effect of BMI on lung volume has been demonstrated $^{(29)}$ in addition to an effect modification of smoking on lung function ${ }^{(30,31)}$. Stratified analysis was conducted by smoking status and BMI strata according to the World Health Organization $^{(32)}$. In addition, the main analyses were repeated with the exclusion of participants with diabetes and/or CVD. Seven confounders contained missing values. In general, missing values were low. The percentages of missing values ranged from 0.02 (height and weight) to $3.4 \%$ (ethnicity). To account for potential attrition bias, multiple imputation was used to create ten different possible copies of the original data set, in which the missing values were substituted by imputed values (online Supplementary Table S1). These imputed values were calculated from their predictive distribution based on the observed data ${ }^{(33)}$. Combined results of the created data sets ( $n$ 10) were then pooled in a separate pooled data set to account for the uncertainty about the missing values. An outline of the procedure is described in the online Supplementary Table S2.

In addition, the main results are presented as effect estimates and $95 \% \mathrm{CI}$ for the indices of lung function, and a $P$ value $<0.05$ was considered statistically significant. IBM SPSS Statistics for Windows (release 21.0.0.1) was used to perform the analyses.

\section{Results}

Table 1 details the study population characteristics, subdivided by cohort. The average age per cohort ranged from 56 to 79 years; women were slightly more represented than men, and the population was almost exclusively Caucasian. The median 
Table 1. Characteristics of participants in the Rotterdam Study ( $n$ 4402)

(Numbers and percentages; mean values and standard deviations; medians and interquartile ranges (IQR))*

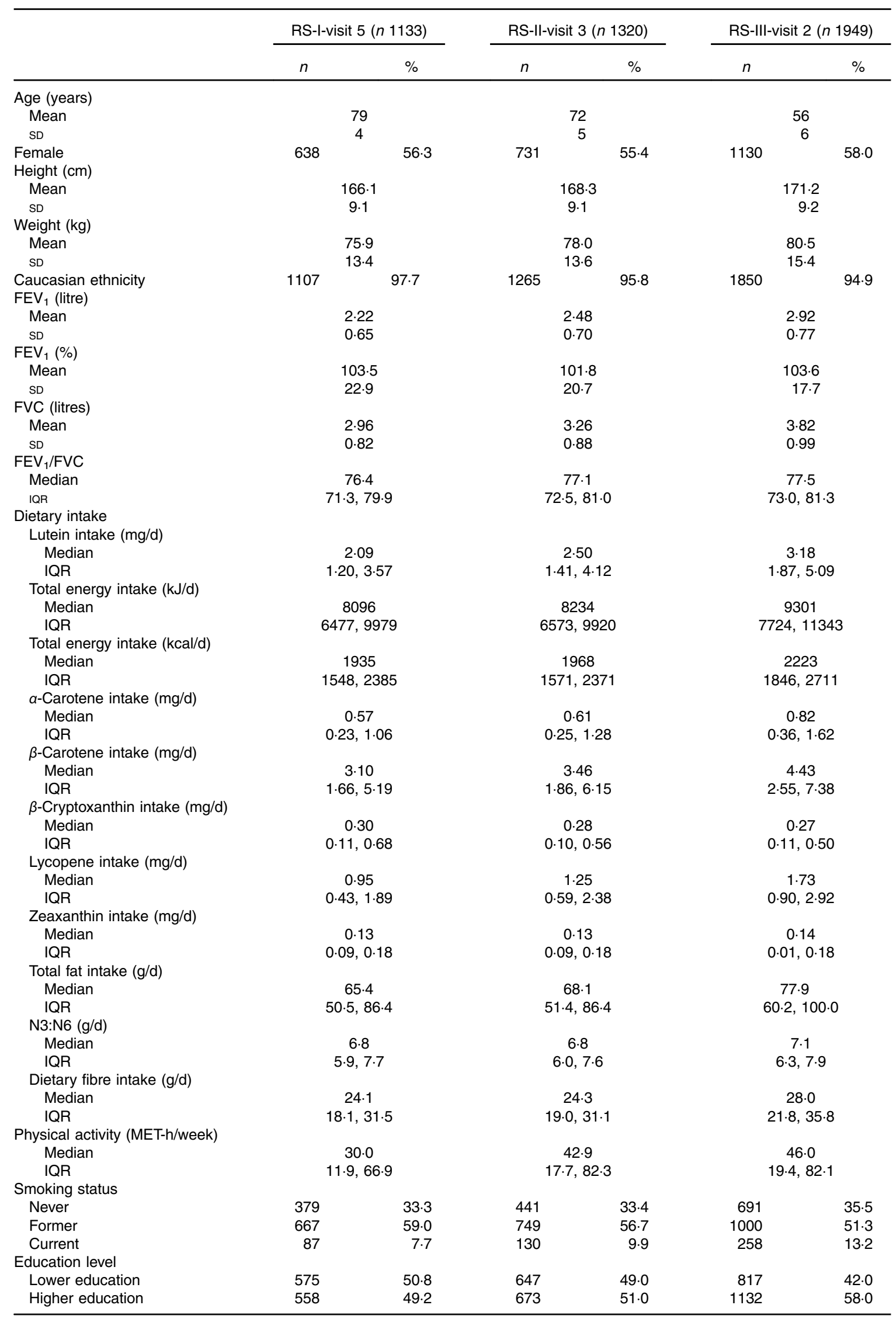


Table 1. Continued

\begin{tabular}{|c|c|c|c|c|c|c|}
\hline & \multicolumn{2}{|c|}{ RS-I-visit 5 ( $n$ 1133) } & \multicolumn{2}{|c|}{ RS-II-visit 3 ( $n$ 1320) } & \multicolumn{2}{|c|}{ RS-III-visit 2 ( $n$ 1949) } \\
\hline & $n$ & $\%$ & $n$ & $\%$ & $n$ & $\%$ \\
\hline Type 2 diabetes mellitus & 137 & $12 \cdot 1$ & 52 & 3.9 & 98 & $5 \cdot 0$ \\
\hline CVD† & 67 & 0.1 & 67 & 5.9 & 63 & $3 \cdot 2$ \\
\hline Asthma & 61 & 5.4 & 75 & $5 \cdot 7$ & 102 & $5 \cdot 2$ \\
\hline COPD & 224 & $19 \cdot 8$ & 211 & $16 \cdot 0$ & 266 & $13 \cdot 6$ \\
\hline Lung cancer & 17 & 1.5 & 11 & 0.8 & 1 & 0.1 \\
\hline
\end{tabular}

$\mathrm{FEV}_{1}$, forced expiratory volume in $1 \mathrm{~s}$; FVC, forced vital capacity; N3:N6, n-3:n-6 fatty acids ratio; MET h/week, metabolic equivalent of task-h per week; COPD, chronic obstructive pulmonary disease.

* Based on imputed data.

† Treatment for narrowed blood vessels, myocardial infarction, stroke, cerebral haemorrhage and cerebrovascular accident.

Table 2. Spearman's correlations of dietary carotenoids ( $n$ 4402)

\begin{tabular}{|c|c|c|c|c|c|c|}
\hline & Lutein & $a$-Carotene & $\beta$-Carotene & $\beta$-Cryptoxanthin & Lycopene & Zeaxanthin \\
\hline Lutein & $\mathrm{x}$ & $0.55^{\star *}$ & $0 \cdot 76^{\star \star}$ & $0.18^{\star \star}$ & $0 \cdot 19^{* *}$ & $0.44^{* *}$ \\
\hline$a$-Carotene & & $\mathrm{x}$ & $0.96^{\star \star}$ & $0.17^{\star \star}$ & $0.19^{\star \star}$ & $0.34^{\star \star}$ \\
\hline$\beta$-Carotene & & & $x$ & $0 \cdot 19^{\star \star}$ & $0.23^{\text {** }}$ & $0.41^{\text {** }}$ \\
\hline$\beta$-Crypotoxanthin & & & & $x$ & $0.13^{\star *}$ & $0.74^{\star *}$ \\
\hline Lycopene & & & & & $\mathrm{x}$ & $0 \cdot 18^{\star \star}$ \\
\hline
\end{tabular}

** $P \leq 0.001$, a $P$ value $<0.05$ is considered to be statistical significant.

daily lutein intake was $2.09 \mathrm{mg} / \mathrm{d}$ in RS-I-visit $5,2.50 \mathrm{mg} / \mathrm{d}$ in RS-II-visit 3 and $3.12 \mathrm{mg} / \mathrm{d}$ in RS-III-visit 1 . The median energy intake was $8101 \mathrm{~kJ} / \mathrm{d}(1935 \mathrm{kcal} / \mathrm{d})$ in RS-I-visit $5,8240 \mathrm{~kJ} / \mathrm{d}$ $(1968 \mathrm{kcal} / \mathrm{d})$ in RS-II-visit 3 and $9307 \mathrm{~kJ} / \mathrm{d}(2223 \mathrm{kcal} / \mathrm{d})$ in RS-III-visit 1. The prevalence of chronic diseases differed among the three cohorts, where 44,32 and $27 \%$ of the participants had diabetes, CVD, COPD, asthma or lung cancer in cohort RS-I-visit 5, RS-II-visit 3 and RS-III-visit 2, respectively. Table 2 shows the Spearman's correlations between the carotenoids. Correlation of lutein with other carotenoids ranged from 0.19 ( $\beta$-cryptoxanthin) to 0.76 ( $\beta$-carotene).

\section{Lutein intake and lung function}

In comparison with the second quartile, the first and lowest quartile of lutein intake was significantly associated with both a lower $\mathrm{FEV}_{1}$ and a lower FVC in the first model adjusted for socio-demographic factors $(-53.07(95 \% \mathrm{CI}-94 \cdot 14,-12 \cdot 01) \mathrm{ml}$, $-49 \cdot 34$ (95\% CI $-95 \cdot 42,-3 \cdot 25) \mathrm{ml}$, respectively) (Table 3). After additional adjustment for lifestyle factors (model 2), the associations were attenuated, and thus they were no longer significant. No significant associations were observed between the third and the fourth quartiles of lutein intake and $\mathrm{FEV}_{1}, \mathrm{FVC}$ and $\mathrm{FEV}_{1} / \mathrm{FVC} \%$, or for the linear associations between lutein and $\mathrm{FEV}_{1}, \mathrm{FVC}$ and $\mathrm{FEV}_{1} / \mathrm{FVC} \%$. Additional adjustment for carotenoids (model 3) did not have an effect on these results.

\section{Sensitivity analysis}

We did not find a significant interaction between lutein and sex, age, lung cancer, asthma or COPD ( $P$ value all $\geq 0 \cdot 133$ ). Although we did not observe a significant interaction for smoking with $\mathrm{FEV}_{1}, \mathrm{FVC}$ or $\mathrm{FEV}_{1} / \mathrm{FVC} \%$ ( $P_{\text {interaction }} \geq 0 \cdot 133$ ), stratified analyses revealed that lutein was significantly associated with lower $\mathrm{FEV}_{1}$ and $\mathrm{FEV}_{1} / \mathrm{FVC} \%$ in smokers after adjusting for socio-demographic factors and lifestyle factors $(-60 \cdot 08$ (95\% CI -115.84, -4.31) ml, -1.64 (95\% CI -2.63, -0.65) \% per SD increase in lutein, respectively) (Tables 4 and 6). After adjustment for other carotenoids, some of the results were attenuated; however, the association remained significant for lutein and FEV1/FVC (-1.69 (95\% CI -2.93, -0.45)\% per SD increase of lutein). As compared with the second quartile, the fourth and highest quartile of lutein intake was significantly associated with a lower $\mathrm{FEV}_{1}$ and a lower FVC in smokers (Tables 4 and 5). Moreover, the association remained significant after full adjustment (model 3: $-157.55 \mathrm{ml}$ fourth quartile $v$. second quartile $(95 \% \mathrm{CI}-311 \cdot 32,-3 \cdot 79)$ ) for $\mathrm{FEV}_{1}$ and attenuated for FVC. Before adjustment for lifestyle factors, lutein was not significantly associated with $\mathrm{FEV}_{1} / \mathrm{FVC} \%$, but the association became significant after adjustment (model 2: $-2 \cdot 79 \%$ fourth quartile $v$. second quartile (95\% CI $-5 \cdot 15,-0 \cdot 41)$ ) (Table 6). After full adjustment, this association became borderline significant.

In addition, we observed similar results after stratification by BMI and exclusion of participants with diabetes and/or CVD (data not shown).

\section{Discussion}

In a population-based prospective cohort study, we investigated the cross-sectional association between dietary lutein intake and lung function as measured by $\mathrm{FEV}_{1}$. We observed a weak association between lutein and $\mathrm{FEV}_{1}$ and FVC in adults aged 45 years and older, after adjustment for sociodemographic factors. However, this was mainly explained by other nutrients and lifestyle factors. Strikingly, we found that lutein intake was associated with lower lung function as measured by $\mathrm{FEV}_{1} / \mathrm{FVC} \%$ in smokers. To date, there are no dietary recommendations regarding lutein intake; therefore, it is important to clarify to what extent lutein may be related to health outcomes. 
Table 3. Association between dietary lutein intake and forced expiratory volume in $1 \mathrm{~s}(\mathrm{FEV})_{1}$, forced vital capacity $(\mathrm{FVC})$ and FEV $/$ /FVC\% $(n 4402)-$ pooled analysis

( $\beta$-Coefficients and $95 \%$ confidence intervals)

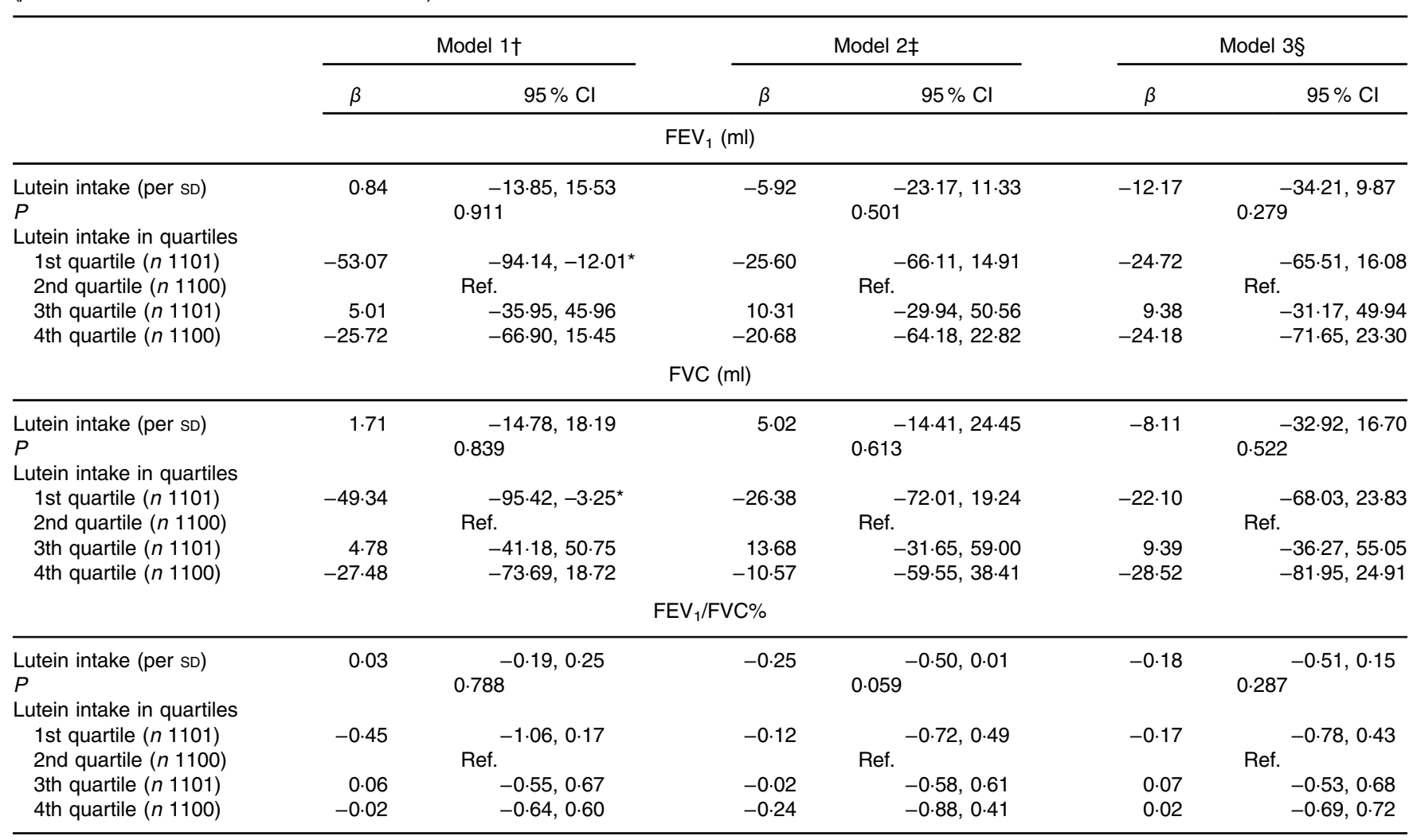

Ref., reference values.

* $P<0.05$.

† Model 1 is adjusted for age, sex, height, ethnicity, cohort and education.

¥ Model 2 is adjusted as for model 1, plus weight, total daily energy intake, total fat intake, $n-3: n-6$ fatty acids ratio, fibre intake, alcohol intake, smoking status and physical activity measured in metabolic equivalent of task-h per week.

$\S$ Model 3 was additionally adjusted for daily intakes of $\beta$-carotene, $\beta$-cryptoxanthin, lycopene and zeaxanthin.

\section{Findings in other studies}

Our finding that lutein is not associated with $\mathrm{FEV}_{1}$ and FVC is in line with a few other observational studies on $\operatorname{FEV}_{1}^{(9,10,15)}$ and one study on $\mathrm{FVC}^{(15)}$. A population-based prospective study found a significant association between lutein/zeaxanthin and $\mathrm{FEV}_{1}, \mathrm{FVC}$ and $\mathrm{FEV}_{1} / \mathrm{FVC} \%$; however, they investigated the association in adults with chronic airflow limitation ${ }^{(14)}$. A previous longitudinal study found a borderline significant association between lutein/zeaxanthin and a slower decline in $\mathrm{FEV}_{1}$, and a significant association between lutein/zeaxanthin and FVC. However, this study was conducted in young adults with a mean age of 25 years ${ }^{(16)}$. A borderline significant association between lutein/zeaxanthin blood levels and FVC has been described ${ }^{(9)}$, and these authors have also demonstrated a significant association between lutein/zeaxanthin intake and $\mathrm{FVC}^{(10)}$. In addition, all studies that investigated lutein/zeaxanthin also investigated the association between other carotenoids and/or antioxidant vitamins and lung function. Schunemann et al..$^{(9,10)}$ reported that lutein/ zeaxanthin dietary intake and blood levels were strongly related to lung function, although not significant for $\mathrm{FEV}_{1}$, as compared with other nutrients with antioxidant capacities such as beta-carotene. This was also reported by Ochs-Balcom et al. ${ }^{(14)}$, who demonstrated that lutein/zeaxanthin, measured by dietary intake and blood levels, was strongly related to $\mathrm{FEV}_{1}$, FVC and $\mathrm{FEV}_{1} / \mathrm{FVC} \%$. Dietary lutein intake has been examined by two out of five studies that investigated lung function in adults $^{(9,10,14-16)}$. A study found a significant positive association between lutein/zeaxanthin and all indices of lung function ${ }^{(14)}$. Moreover, a second study found a significantly positive association between lutein/zeaxanthin and FVC only ${ }^{(10)}$.

Interestingly, subgroup analysis by smoking status in our study revealed that lutein intake was associated with a lower lung function as measured by $\mathrm{FEV}_{1} / \mathrm{FVC} \%$. Although this has not been reported before, other studies have documented the potential harmful effects of other carotenoids on lung outcomes. For example, intervention studies suggest that carotenoid supplementation increased lung cancer and mortality in heavy smokers ${ }^{(34-38)}$. Indeed, the association between lutein intake and $\mathrm{FEV}_{1}$ in smokers was explained by adjustment for other carotenoids, suggesting that the inverse association between lutein intake and indices of lung function may be explained to a certain extent by other carotenoids. 
Table 4. Association between dietary lutein intake and forced expiratory volume in $1 \mathrm{~s}\left(\mathrm{FEV}_{1}\right)$, stratified by smoking status - pooled analysis ( $\beta$-Coefficients and $95 \%$ confidence intervals)

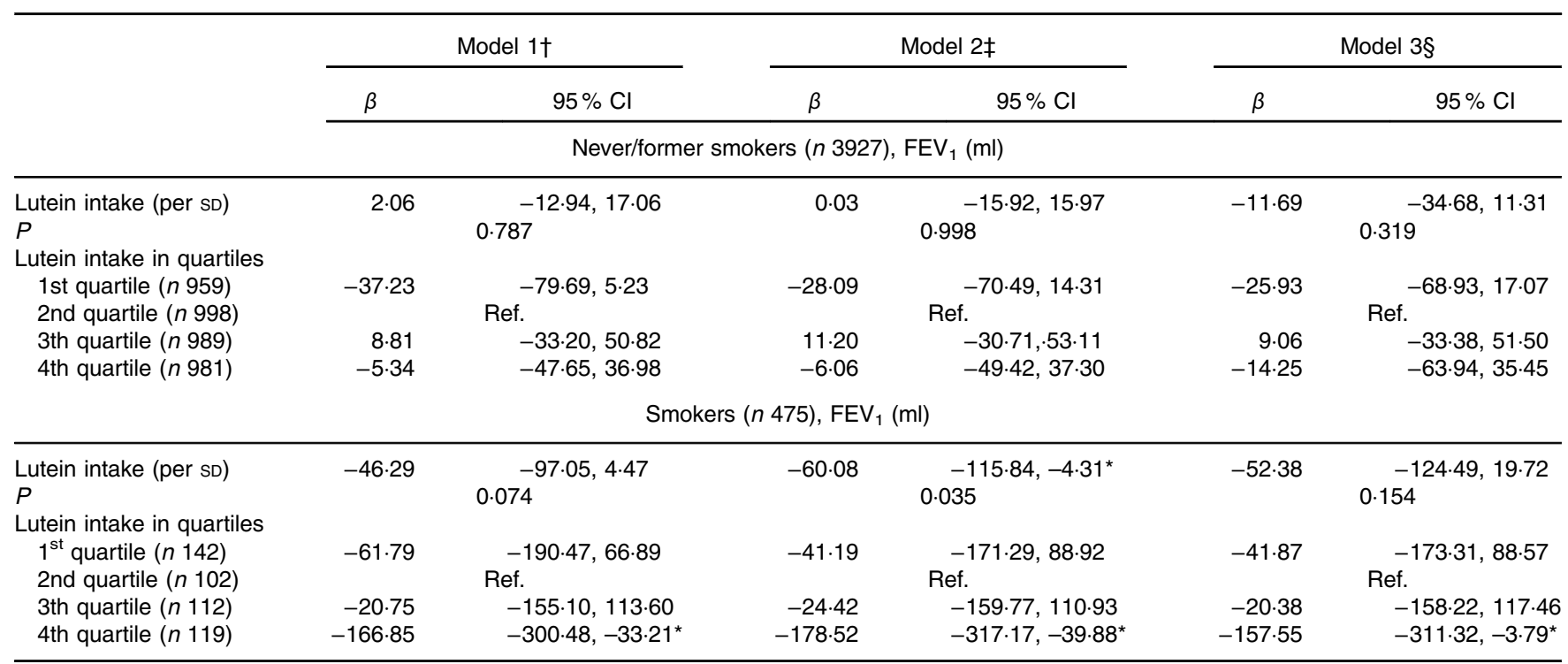

Ref., reference values.

${ }^{*} P<0.05\left(P_{\text {interaction }}=0.13\right)$.

† Model 1 is adjusted for age, sex, height, ethnicity, cohort and education.

$\ddagger$ Model 2 is adjusted as for model 1 , plus weight, total daily energy intake, total fat intake, $n-3: n-6$ fatty acids ratio, fibre intake, alcohol intake and physical activity measured in metabolic equivalent of task-h per week.

$\S$ Model 3 was additionally adjusted for daily intakes of $\beta$-carotene, $\beta$-cryptoxanthin, lycopene and zeaxanthin.

Table 5. Association between dietary lutein intake and forced vital capacity (FVC), stratified by smoking status - pooled analysis ( $\beta$-Coefficients and $95 \%$ confidence intervals)

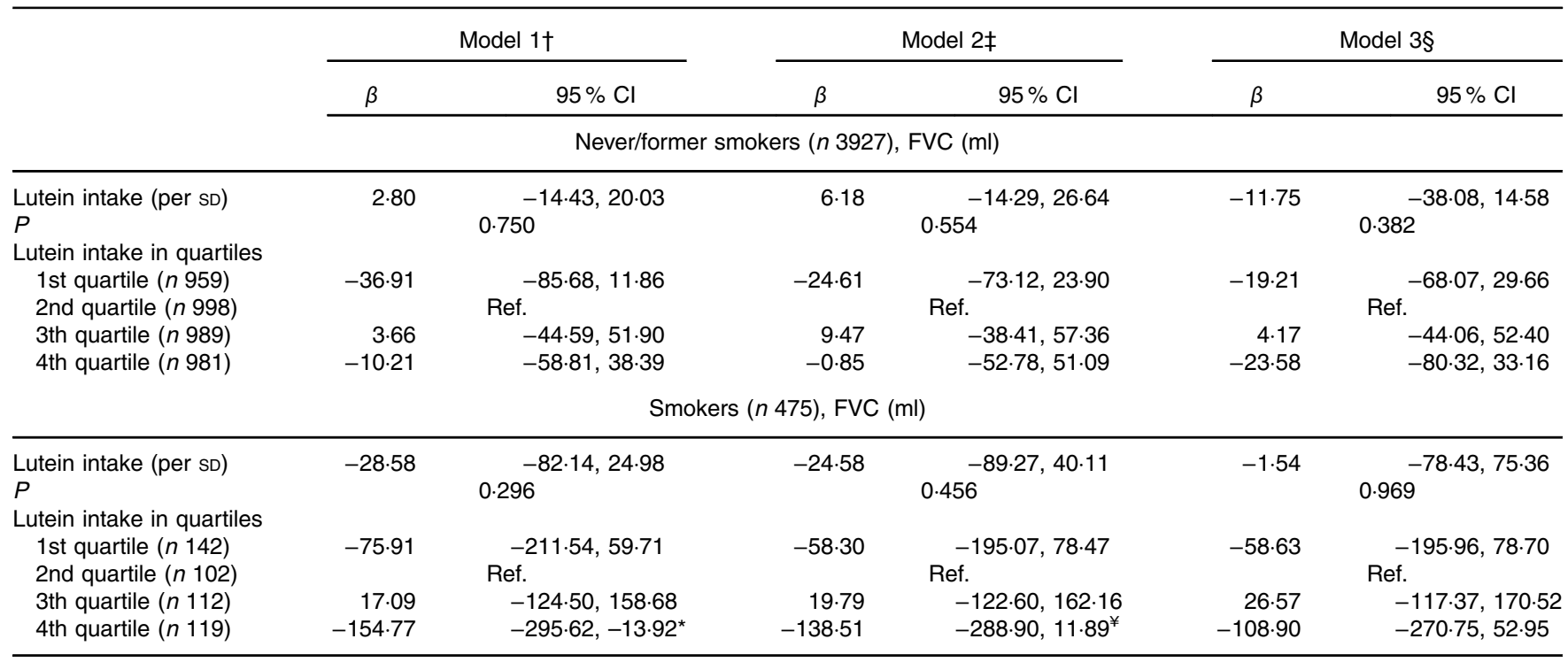

Ref., reference values.

$P_{\text {interaction }}=0.549,{ }^{*} P<0.05,{ }^{¥} P=0.071$.

$\dagger$ Model 1 is adjusted for age, sex, height, ethnicity, cohort and education.

‡ Model 2 is adjusted as for model 1 , plus weight, total daily energy intake, total fat intake, $n-3: n-6$ fatty acids ratio, fibre intake, alcohol intake and physical activity measured in metabolic equivalent of task-h per week.

$\S$ Model 3 was additionally adjusted for daily intakes of $\beta$-carotene, $\beta$-cryptoxanthin, lycopene and zeaxanthin.

\section{Mechanisms}

The belief that relates lutein intake with improved respiratory health is based mainly on counteracting oxidative stress.
Antioxidants are known to act against reactive oxygen species (ROS), which can be divided into exogeneous oxidants (e.g. cigarette smoke) and endogeneous oxidants (i.e. produced by inflammatory cells $)^{(39,40)}$. Oxidative stress, an imbalance 
Table 6. Association between dietary lutein intake and forced expiratory volume in $1 \mathrm{~s}\left(\mathrm{FEV}_{1}\right) /$ forced vital capacity $(\mathrm{FVC} \%)$, stratified by smoking status - pooled analysis

( $\beta$-Coefficients and $95 \%$ confidence intervals)

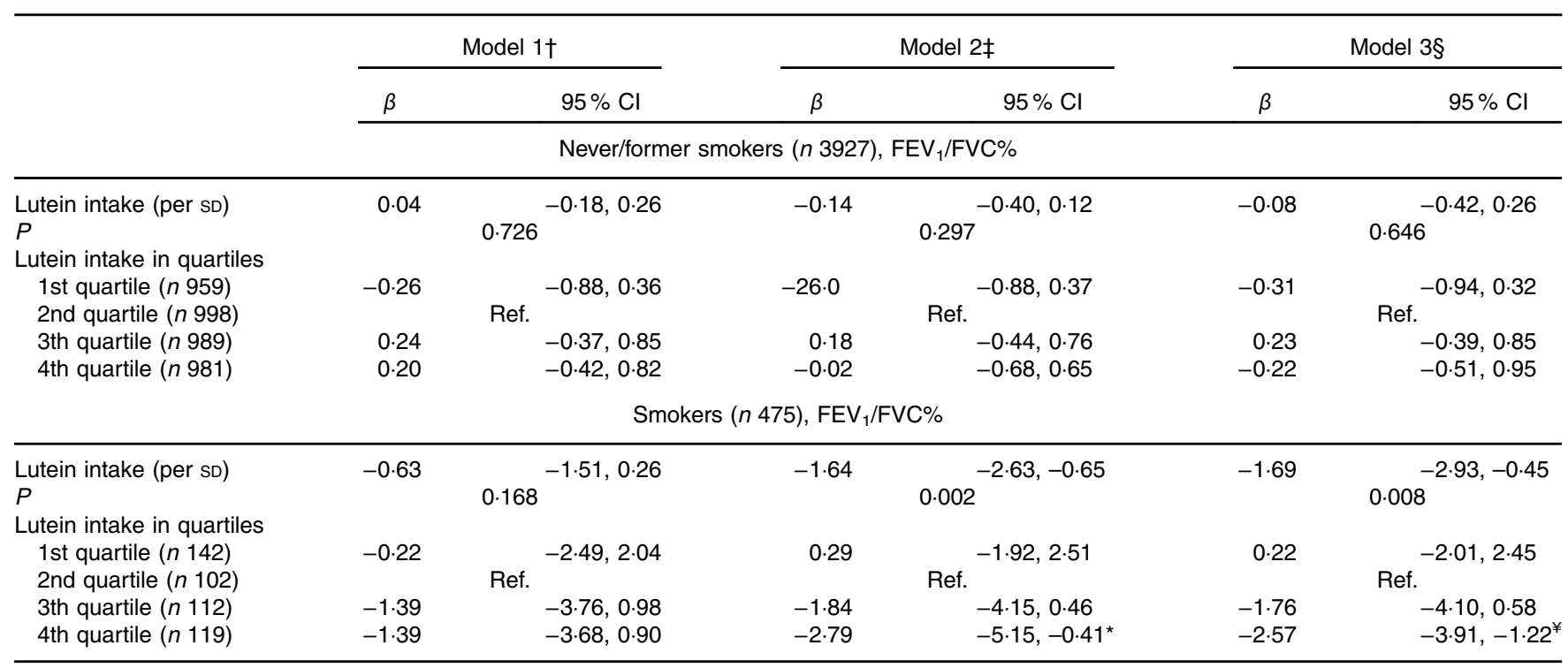

Ref., reference values.

$P_{\text {interaction }}=0.033,{ }^{*} P<0.05,{ }^{*} P=0.056$.

$\dagger$ Model 1 is adjusted for age, sex, height, ethnicity, cohort and education.

$\ddagger$ Model 2 is adjusted as for model 1, plus weight, total daily energy intake, total fat intake, $n-3: n-6$ fatty acids ratio, fibre intake, alcohol intake and physical activity measured in metabolic equivalent of task-h per week.

$\S$ Model 3 was additionally adjusted for daily intake of $\beta$-carotene, $\beta$-cryptoxanthin, lycopene and zeaxanthin.

between antioxidant capacity and $\operatorname{ROS}^{(41,42)}$, is suggested to be associated with worsening of lung function. For instance, an association between increased markers of oxidative stress (i.e. malondialdehyde or oxidised LDL and decreased lung function in COPD patients has been found ${ }^{(43,44)}$. However, our results are not in line with this hypothesis. On the basis of this mechanism, it can be suggested that the function of lutein (as an antioxidant) depends on the level of exposure to smoking (as an oxidant). For that reason, we performed stratified analyses by smoking status, as it has been proposed that a high intake of carotenoids may reduce their protective function against ROS, particularly in smokers and may even enhance smoke-induced oxidative stress ${ }^{(45)}$. A possible explanation for this lost function is that cigarette smoke modifies the chemical composition of these nutrients, turning antioxidants into pro-oxidants ${ }^{(46)}$. Hence, further studies on the potentially harmful effects of dietary lutein intake in smokers are required.

\section{Methodological considerations}

Several important strengths of our study can be acknowledged. In comparison with previous studies, we have a large population-based sample size, and were able to adjust for a wide range of confounders including lifestyle and dietary factors as well as intakes of other carotenoids, which were found to be very important.

However, some limitations also need to be taken into account. First, our study had an observational cross-sectional design, which prevents final conclusions about the causality of the observed associations. We found that other dietary and lifestyle factors largely explained the observed association. With this in mind, it may be important to study overall dietary patterns in relation to lung function as this takes the intercorrelation between dietary factors into account ${ }^{(47)}$. Indeed, several dietary patterns have been found to be associated with lung function ${ }^{(48-54)}$. Second, we only had information on dietary intake of lutein and not on blood levels. It may be argued that the blood levels of lutein give a better reflection of lutein status in the human body. For example, when studying blood levels, unmeasured factors such as genetic factors can be better taken into account ${ }^{(55)}$. In contrast, when high oxidative stress is present, such as in COPD, blood levels of antioxidants may decrease because of an increased demand, which leads to lower blood levels of antioxidants as a consequence, such as lutein ${ }^{(56)}$. Hence, reverse causality might be present, as it remains unknown whether individuals with, for example, COPD have low blood levels of antioxidants as a consequence of the disease (e.g. physiologically or due to altered dietary intake), or whether low blood levels contribute to the development of the disease. Although our results were not different after excluding participants with chronic diseases, reverse causation might still partly explain the findings. Third, we used a FFQ for dietary assessment of lutein, which is subject to measurement error. To account for potential systematic measurement error, we adjusted lutein intake for total energy intake ${ }^{(28)}$. However, non-differential misclassification may still be present, which may have led to bias towards the null ${ }^{(57)}$. Fourth, selection bias or survival bias may be present, as we selected participants for this study on the basis of available information on their dietary intakes and lung function. 
Individuals with lower lung function or at a severe stage of chronic disease might not have visited the research centre, and therefore may have been excluded from the present study. This exclusion could have underestimated our association. However, the socio-demographic characteristics (e.g. age, sex, height, body weight, ethnicity and smoking status) of the included participants in our study did not differ from the total population of the Rotterdam Study ${ }^{(58)}$.

In conclusion, we investigated the cross-sectional association between dietary lutein intake and lung function in a large, population-based, prospective cohort study. Although we observed some associations between lutein intake and lung function, the majority of these disappeared after adjustment for other nutrients and lifestyle factors. This suggests that a combination of healthy dietary and lifestyle factors might contribute to an improved lung function instead of lutein intake alone. Future studies in the general population should focus on whole diet to identify patterns in foods linked to specific nutrients that are associated with lung function. Interestingly, we observed that higher lutein intake was associated with a lower lung function in smokers. This finding supports previous evidence on the adverse effect of high intakes of antioxidants in smokers. However, to date, there is no dietary recommendation for lutein, which is urgently needed in the clinical setting, as harmful effects might manifest when a high dose is taken by particular risk groups. Future studies should also be aware of the potential inverse association between high lutein intake and specific risk groups such as smokers. To date, the effect of high doses of nutrients that are included in nutritional supplements for the general population or risk groups is poorly studied. A trial by Omenn et al. ${ }^{(38)}$ investigated the protective effect of high doses of vitamin $\mathrm{A}$ and $\beta$-carotene on lung cancer, which were discontinued when the incident risk of lung cancer was shown to be higher in the treatment group as compared with the control group. Some trials supported their findings, and some other research groups did not ${ }^{(59)}$. In addition, some risk groups might benefit from a nutrition supplement and others may not ${ }^{(59)}$. For example, (pre) pregnant women are advised to take folate supplementation to avoid spina bifida; however, a high dose of folate increased the risk of adenomas in a trial investigating colorectal adenomas ${ }^{(60)}$. These examples demonstrate that more research is needed to give a public health recommendation on nutritional supplements.

\section{Supplementary material}

For supplementary material/s referred to in this article, please visit https:/doi.org/10·1017/S0007114517000319

\section{Acknowledgements}

The authors gratefully thank Roisin McManus for revising the grammar of the manuscript.

The Rotterdam Study is funded by Erasmus MC and Erasmus University, Rotterdam, The Netherlands; the Netherlands Organization for the Health Research and Development (ZonMw); the Research Institute for Diseases in the Elderly
(RIDE); the Ministry of Education, Culture and Science, the Ministry for Health, Welfare and Sports; the European Commission (DG XII); and the Municipality of Rotterdam.

D. M. v. L., E. T. M. L., J. C. K.-d. J., L. L. and O. H. F. designed the study. D. M. v. L., conducted the study. A. H., O. H. F., G. G. B. and B. H. S. provided essential materials. D. M. v. L. and E. T. M. L. analysed the data. D. M. v. L, E. T. M. L., L. L. and J. C. K.-d. J. wrote the manuscript. L. L. and J. C. K.-d. J. had primary responsibility for the final content. A. H., B. H. S., G. G. B. and O. H. F. contributed to the intellectual content while drafting the manuscript. All the authors read and approved the final version of the manuscript.

D. M. v. L., E. T. M. L., J. C. K.-d. J. and O. H. F. work in ErasmusAGE, a centre for ageing research across the life course funded by Nestlé Nutrition (Nestec Ltd), Metagenics Inc. and AXA. Nestlé Nutrition (Nestec Ltd), Metagenics Inc. and AXA had no role in the design and conduct of the study; collection, management, analysis and interpretation of the data as well as preparation, review or approval of the manuscript. L. L. is a Postdoctoral Fellow of the Research Foundation Flanders (FWO).

\section{References}

1. Lozano R, Naghavi M, Foreman K, et al. (2012) Global and regional mortality from 235 causes of death for 20 age groups in 1990 and 2010: a systematic analysis for the Global Burden of Disease Study 2010. Lancet 380, 2095-2128.

2. Sharma G \& Goodwin J (2006) Effect of aging on respiratory system physiology and immunology. Clin Interv Aging $\mathbf{1}$, 253-260.

3. Friedman GD, Klatsky AL \& Siegelaub AB (1976) Lung function and risk of myocardial infarction and sudden cardiac death. N Engl J Med 294, 1071-1075.

4. Schunemann HJ, Dorn J, Grant BJ, et al. (2000) Pulmonary function is a long-term predictor of mortality in the general population: 29-year follow-up of the Buffalo Health Study. Chest 118, 656-664.

5. Itoh M, Tsuji T, Nemoto K, et al. (2013) Undernutrition in patients with COPD and its treatment. Nutrients $\mathbf{5}$, 1316-1335

6. Hanson C, Rutten EP, Wouters EF, et al. (2014) Influence of diet and obesity on COPD development and outcomes. Int J Chron Obstruct Pulmon Dis 9, 723-733.

7. Kijlstra A, Tian Y, Kelly ER, et al. (2012) Lutein: more than just a filter for blue light. Prog Retin Eye Res 31, 303-315.

8. US Department of Agriculture and Agricultural Research Service (2011) USDA National Nutrient Database for Standard Reference. http://ndb.nal.usda.gov/ (accessed July 2015).

9. Schunemann HJ, Grant BJ, Freudenheim JL, et al. (2001) The relation of serum levels of antioxidant vitamins $\mathrm{C}$ and $\mathrm{E}$, retinol and carotenoids with pulmonary function in the general population. Am J Respir Crit Care Med 163, $1246-1255$.

10. Schunemann HJ, McCann S, Grant BJ, et al. (2002) Lung function in relation to intake of carotenoids and other antioxidant vitamins in a population-based study. Am J Epidemiol 155, 463-471.

11. Keranis E, Makris D, Rodopoulou P, et al. (2010) Impact of dietary shift to higher-antioxidant foods in COPD: a randomised trial. Eur Respir J 36, 774-780. 
12. Tsiligianni IG \& van der Molen T (2010) A systematic review of the role of vitamin insufficiencies and supplementation in COPD. Respir Res 11, 171.

13. Melo van Lent D, Leermakers ETM, Darweesh SKL, et al. (2016) The effects of lutein on respiratory health across the life course: a systematic review. Clin Nutr ESPEN 13, e1-e7.

14. Ochs-Balcom HM, Grant BJ, Muti P, et al. (2006) Antioxidants, oxidative stress, and pulmonary function in individuals diagnosed with asthma or COPD. Eur I Clin Nutr 60 , 991-999.

15. Semba RD, Chang SS, Sun K, et al. (2012) Serum carotenoids and pulmonary function in older communitydwelling women. J Nutr Health Aging 16, 291-296.

16. Thyagarajan B, AM K, Smith LJ, et al. (2011) Serum carotenoid concentrations predict lung function evolution in young adults: the Coronary Artery Risk Development in Young Adults (CARDIA) study. Am J Clin Nutr 94, 1211-1218.

17. Ikram MA, van der Lugt A, Niessen WJ, et al. (2015) The Rotterdam Scan Study: design update 2016 and main findings. Eur J Epidemiol 30, 1299-1315.

18. Oeppen J \& Vaupel JW (2002) Demography. Broken limits to life expectancy. Science 296, 1029-1031.

19. Feunekes GI, Van Staveren WA, De Vries JH, et al. (1993) Relative and biomarker-based validity of a food-frequency questionnaire estimating intake of fats and cholesterol. $\mathrm{Am} \mathrm{J}$ Clin Nutr 58, 489-496.

20. Goldbohm RA, van den Brandt PA, Brants HA, et al. (1994) Validation of a dietary questionnaire used in a large-scale prospective cohort study on diet and cancer. Eur J Clin Nutr 48, 253-265.

21. Donders-Engelen M \& van der Heijden L (2003) Maten, gewichten en codenummers 2003. Wageningen and Zeist: Wageningen UR and Vakgroep Humane Voeding and TNO Voeding.

22. Westenbrink S, Jansen-van der Vliet M, Brants HAM, et al. (2006) NEVO-tabel Nederlands voedingsstoffenbestand. Utrecht: Hoontetijl, Stichting NEVO 2006.

23. Voedingscentrum Rijksinstituut voor Volksgezondheid en Milieu (RIVM) (2011) Nederlandse Voedingsmiddelen Tabel (NEVO-tabel 2011), 1st ed. Den Haag: Voedingscentrum RIVM.

24. Celli BR, MacNee W \& Force AET (2004) Standards for the diagnosis and treatment of patients with COPD: a summary of the ATS/ERS position paper. Eur Respir J 23, 932-946.

25. NErler (2015) NErler/shiny-nonlinear. https://github.com/ NErler/shiny-nonlinear (accessed July 2015).

26. Johnson EJ (2002) The role of carotenoids in human health. Nutr Clin Care 5, 56-65.

27. Mickey RM \& Greenland S (1989) The impact of confounder selection criteria on effect estimation. Am J Epidemiol 129, 125-137.

28. Willett WC, Howe GR \& Kushi LH (1997) Adjustment for total energy intake in epidemiologic studies. Am J Clin Nutr $\mathbf{6 5}$ 1220S-1228S ; discussion 1229S-1231S.

29. Jones RL \& Nzekwu MM (2006) The effects of body mass index on lung volumes. Chest 130, 827-833.

30. Larose TL, Brumpton BM, Langhammer A, et al. (2015) Serum 25-hydroxyvitamin D level, smoking and lung function in adults: the HUNT Study. Eur Respir J 46, 355-363.

31. Lange NE, Sparrow D, Vokonas P, et al. (2012) Vitamin D deficiency, smoking, and lung function in the Normative Aging Study. Am J Respir Crit Care Med 186, 616-621.

32. World Health Organization (2015) Body mass index - BMI http://www.euro.who.int/en/health-topics/disease-prevention/ nutrition/a-healthy-lifestyle/body-mass-index-bmi (accessed July 2015).
33. Sterne JA, White IR, Carlin JB, et al. (2009) Multiple imputation for missing data in epidemiological and clinical research: potential and pitfalls. BMJ 338, b2393.

34. Slatore CG, Littman AJ, Au DH, et al. (2008) Long-term use of supplemental multivitamins, vitamin $\mathrm{C}$, vitamin $\mathrm{E}$, and folate does not reduce the risk of lung cancer. Am J Respir Crit Care Med 177, 524-530.

35. Gabriel HE, Liu Z, Crott JW, et al. (2006) A comparison of carotenoids, retinoids, and tocopherols in the serum and buccal mucosa of chronic cigarette smokers versus nonsmokers. Cancer Epidemiol Biomarkers Prev 15, 993-999.

36. Greenwald P, Anderson D, Nelson SA, et al. (2007) Clinical trials of vitamin and mineral supplements for cancer prevention. Am J Clin Nutr 85, 314S-317S

37. Satia JA, Littman A, Slatore CG, et al. (2009) Long-term use of beta-carotene, retinol, lycopene, and lutein supplements and lung cancer risk: results from the VITamins And Lifestyle (VITAL) study. Am J Epidemiol 169, 815-828.

38. Omenn GS, Goodman GE, Thornquist MD, et al. (1996) Effects of a combination of beta carotene and vitamin $\mathrm{A}$ on lung cancer and cardiovascular disease. $N$ Engl J Med 334, 1150-1155.

39. Halliwell B (1990) How to characterize a biological antioxidant. Free Radic Res Commun 9, 1-32.

40. Genestra M (2007) Oxyl radicals, redox-sensitive signalling cascades and antioxidants. Cell Signal 19, 1807-1819.

41. MacNee W (2000) Oxidants/antioxidants and COPD. Chest 117, 303S-317S.

42. Schunemann HJ, Freudenheim JL \& Grant BJ (2001) Epidemiologic evidence linking antioxidant vitamins to pulmonary function and airway obstruction. Epidemiol Rev 23, 248-267.

43. Shen Y, Yang T, Guo S, et al. (2013) Increased serum ox-LDL levels correlated with lung function, inflammation, and oxidative stress in COPD. Mediators Inflamm 2013, 972347.

44. Ahmad A, Shameem M \& Husain Q (2013) Altered oxidantantioxidant levels in the disease prognosis of chronic obstructive pulmonary disease. Int J Tuberc Lung Dis 17, 1104-1109.

45. Palozza P, Serini S, Trombino S, et al. (2006) Dual role of betacarotene in combination with cigarette smoke aqueous extract on the formation of mutagenic lipid peroxidation products in lung membranes: dependence on pO2. Carcinogenesis 27, 2383-2391.

46. Hurst JS, Contreras JE, Siems WG, et al. (2004) Oxidation of carotenoids by heat and tobacco smoke. Biofactors 20, 23-35.

47. Hu FB (2002) Dietary pattern analysis: a new direction in nutritional epidemiology. Curr Opin Lipidol 13, 3-9.

48. Butland BK, Fehily AM \& Elwood PC (2000) Diet, lung function, and lung function decline in a cohort of 2512 middle aged men. Thorax 55, 102-108.

49. Tabak C, Smit HA, Heederik D, et al. (2001) Diet and chronic obstructive pulmonary disease: independent beneficial effects of fruits, whole grains, and alcohol (the MORGEN study). Clin Exp Allergy 31, 747-755.

50. Kelly Y, Sacker A \& Marmot M (2003) Nutrition and respiratory health in adults: findings from the health survey for Scotland. Eur Respir J 21, 664-671.

51. Varraso R, Chiuve SE, Fung TT, et al. (2015) Alternate Healthy Eating Index 2010 and risk of chronic obstructive pulmonary disease among US women and men: prospective study. BMJ 350, h286.

52. Shaheen SO, Jameson KA, Syddall HE, et al. (2010) The relationship of dietary patterns with adult lung function and COPD. Eur Respir J 36, 277-284.

53. Varraso R, Fung TT, Barr RG, et al. (2007) Prospective study of dietary patterns and chronic obstructive pulmonary disease among US women. Am J Clin Nutr 86, 488-495. 
54. Varraso R, Fung TT, Hu FB, et al. (2007) Prospective study of dietary patterns and chronic obstructive pulmonary disease among US men. Thorax 62, 786-791.

55. Gruber M, Chappell R, Millen A, et al. (2004) Correlates of serum lutein + zeaxanthin: findings from the Third National Health and Nutrition Examination Survey. J Nutr 134, 2387-2394.

56. Ford ES, Mokdad AH, Giles WH, et al. (2003) The metabolic syndrome and antioxidant concentrations: findings from the Third National Health and Nutrition Examination Survey. Diabetes 52, 2346-2352.
57. Rothman KJ (2002) Epidemiology - An Introduction. New York: Oxford University Press.

58. Terzikhan N, Verhamme KM, Hofman A, et al. (2016) Prevalence and incidence of COPD in smokers and non-smokers: the Rotterdam Study. Eur J Epidemiol 31, 785-792.

59. Goralczyk R (2009) Beta-carotene and lung cancer in smokers: review of hypotheses and status of research. Nutr Cancer $\mathbf{6 1}$, 767-774.

60. Cole BF, Baron JA, Sandler RS, et al. (2007) Folic acid for the prevention of colorectal adenomas: a randomized clinical trial. JAMA 297, 2351-2359. 